\title{
Partial ovoids and partial spreads in hermitian polar spaces
}

\author{
J. De Beule • A. Klein • K. Metsch • L. Storme
}

Received: 26 September 2006 / Revised: 12 January 2007 /

Accepted: 2 February 2007

(C) Springer Science+Business Media, LLC 2007

\begin{abstract}
We present improved lower bounds on the sizes of small maximal partial ovoids in the classical hermitian polar spaces, and improved upper bounds on the sizes of large maximal partial spreads in the classical hermitian polar spaces. Of particular importance is the presented upper bound on the size of a maximal partial spread of $H\left(3, q^{2}\right)$. For $q=2,3$, the presented upper bound is sharp. For $q=3$, our results confirm via theoretical arguments properties, deduced by computer searches performed by Ebert and Hirschfeld, for the largest partial spreads of $H(3,9)$. An overview of the status regarding these results is given in two summarizing tables. The similar results for the classical symplectic and orthogonal polar spaces are presented in De Beule et al. [8].
\end{abstract}

Keywords Hermitian variety $\cdot$ Polar space $\cdot$ Partial ovoid $\cdot$ Partial spread

AMS Classifications $\quad 05 \mathrm{~B} 25 \cdot 51 \mathrm{D} 20 \cdot 52 \mathrm{C} 17 \cdot 52 \mathrm{C} 35$

\section{Introduction}

This article is the second of two articles discussing partial ovoids and partial spreads in finite classical polar spaces. Here, we discuss the hermitian polar spaces. In De Beule et al. [8], we discussed the symplectic and orthogonal polar spaces.

J. De Beule $(\bowtie) \cdot$ A. Klein · L. Storme

Department of Pure Mathematics and Computer Algebra, Ghent University,

Krijgslaan 281-S22, 9000 Ghent, Belgium

e-mail: jdebeule@cage.ugent.be

A. Klein

e-mail: klein@cage.ugent.be

K. Metsch

Mathematisches Institut, Justus-Liebig-Universität, Arndtstrasse 2, 35392 Giessen, Germany e-mail: Klaus.Metsch@math.uni-giessen.de

L. Storme

e-mail: ls@cage.ugent.be 
The classical finite polar spaces are the non-singular symplectic polar spaces $W(2 n+$ $1, q)$, the non-singular parabolic quadrics $Q(2 n, q), n \geq 2$, the non-singular elliptic and hyperbolic quadrics $Q^{-}(2 n+1, q), n \geq 2$, and $Q^{+}(2 n+1, q), n \geq 1$, and the non-singular hermitian varieties $H\left(d, q^{2}\right), d \geq 3$. For $q$ even, the parabolic polar spaces $Q(2 n, q)$ are isomorphic to the symplectic polar spaces $W(2 n-1, q)$.

The generators of a classical polar space are the subspaces of maximal dimension contained in these polar spaces. If the generators are of dimension $r-1$, then the polar space is said to be of rank $r$.

The polar spaces of rank $r=2$ coincide with the generalized quadrangles.

The finite classical generalized quadrangles are the non-singular parabolic quadric $Q(4, q)$, the non-singular elliptic quadric $Q^{-}(5, q)$, the non-singular hyperbolic quadric $Q^{+}(3, q)$, the non-singular hermitian varieties $H\left(3, q^{2}\right)$ and $H\left(4, q^{2}\right)$, and the symplectic generalized quadrangle $W(3, q)$ in $P G(3, q)$. The generalized quadrangles $Q(4, q)$ and $W(3, q)$ are dual to each other. The generalized quadrangles $Q(4, q)$ and $W(3, q)$ are self-dual if and only if $q$ is even. Finally, $H\left(3, q^{2}\right)$ and $Q^{-}(5, q)$ also are dual to each other.

An ovoid of a classical polar space $\mathcal{P}$ is a set $\mathcal{O}$ of points of $\mathcal{P}$ such that every generator contains exactly one point of $\mathcal{O}$. A partial ovoid of a classical polar space $\mathcal{P}$ is a set $\mathcal{O}$ of points of $\mathcal{P}$ such that every generator contains at most one point of $\mathcal{O}$. A spread of a classical polar space $\mathcal{P}$ is a set $\mathcal{S}$ of generators of $\mathcal{P}$ partitioning the point set of $\mathcal{P}$. A partial spread of a classical polar space $\mathcal{P}$ is a set $\mathcal{S}$ of pairwise disjoint generators of $\mathcal{P}$. A partial ovoid or partial spread is called maximal when it is not contained in a larger partial ovoid or partial spread of the same polar space.

Let $X:=|\mathcal{P}| /|\Pi|$, where $\Pi$ is a generator of $\mathcal{P}$. Then $X$ is the size of an ovoid or spread in $\mathcal{P}$, in case $\mathcal{P}$ effectively contains an ovoid or spread. Assume that $\mathcal{O}$ is a partial spread or partial ovoid of $\mathcal{P}$, then $X-|\mathcal{O}|$ is called the deficiency of $\mathcal{O}$.

The first natural problem regarding ovoids and spreads in finite classical polar spaces is that of the existence of these ovoids and spreads [12,18,19].

Then research was focused on the size of the largest partial ovoids and spreads of finite classical polar spaces that do not have ovoids or spreads, and to the problem of the extendability of partial ovoids and partial spreads to ovoids and spreads when the finite classical polar spaces have ovoids and spreads $[11,18]$.

Recently, attention was also paid to the problem of the cardinality of the smallest maximal partial ovoids and the smallest maximal partial spreads in finite classical generalized quadrangles and polar spaces $[1,2,6,7,10,15,16]$. In particular, $[13,16]$ addressed these problems for the classical generalized quadrangles.

We now present a large number of results on the smallest maximal partial ovoids, and on large maximal partial spreads, for the finite classical hermitian polar spaces. For the analogous results for the classical orthogonal and symplectic polar spaces, we refer to [8]. We conclude the article with two tables presenting the present status on these problems.

\section{Small maximal partial ovoids}

When working in a hermitian polar space $H\left(d, q^{2}\right)$, we denote by $\perp$ the related polarity of the ambient projective space $\operatorname{PG}\left(d, q^{2}\right)$. Two subspaces $\pi, \tau$ with $\tau \subseteq \pi^{\perp}$ are called perpendicular. 
We now focus on small maximal partial ovoids of hermitian polar spaces. The following results on the polar spaces $H\left(3, q^{2}\right)$ and $H\left(4, q^{2}\right)$ are known.

\section{Result 2.1}

(a) [2] If $q$ is even, then a maximal partial ovoid of $H\left(3, q^{2}\right)$ has size at least $q^{2}+1$, and examples of that size exist.

(b) [15] If $q$ is even, then there are no maximal partial ovoids of $H\left(3, q^{2}\right)$ having size in the interval $\left[q^{2}+2, q^{2}+1+4 q / 9\right]$.

(c) [15] A maximal partial ovoid of $H\left(3, q^{2}\right)$, q odd, has size at least $q^{2}+1+4 q / 9$.

(d) [16] A maximal partial ovoid of $H\left(4, q^{2}\right)$ has size at least $q^{2}+q+2$.

We now focus on small maximal partial ovoids of $H\left(2 n, q^{2}\right), n \geq 3$, and of $H(2 n+$ $\left.1, q^{2}\right), n \geq 2$. These are the hermitian polar spaces of rank at least three.

We first discuss $H\left(2 n, q^{2}\right), n \geq 3$, and show that maximal partial ovoids of $H\left(2 n, q^{2}\right)$, $n \geq 3$, contain at least $q^{2}+q+1$ points.

Theorem 2.2 A maximal partial ovoid of $H\left(2 n, q^{2}\right), n \geq 3$, contains at least $q^{2}+q+1$ points.

Proof Assume that $\mathcal{O}$ is a maximal partial ovoid of $H\left(2 n, q^{2}\right), n \geq 3$, of size at most $q^{2}+q$.

Let $\pi$ be a free generator with respect to $\mathcal{O}$, that is a generator missing $\mathcal{O}$. Then the tangent hyperplane $P^{\perp}$ to a point $P$ of $\mathcal{O}$ intersects $\pi$ in a hyperplane of $\pi$. Since $\mathcal{O}$ is a maximal partial ovoid of $H\left(2 n, q^{2}\right)$, the hyperplanes $P^{\perp} \cap \pi, P \in \mathcal{O}$, cover all points of $\pi$; in other words, they form a dual blocking set of $\pi$, see [5].

The smallest dual blocking set of $\pi$ consists of the $q^{2}+1$ hyperplanes of $\pi$ through a fixed ( $n-3)$-dimensional space of $\pi$ [4]. The theory of blocking sets learns us that the smallest dual blocking set of $\pi$ not containing all hyperplanes of $\pi$ through a common $(n-3)$-dimensional space is a dual Baer subplane of size $q^{2}+q+1$, see [5].

So, since $|\mathcal{O}| \leq q^{2}+q$, there is an $(n-3)$-dimensional space $\pi_{1}^{\prime}$ of $\pi$ lying in a pencil of $q^{2}+1$ hyperplane intersections $P^{\perp} \cap \pi, P \in \mathcal{O}$.

Consider the polar space $\pi_{1}^{\prime \perp}$ of $\pi_{1}^{\prime}$ with respect to $H\left(2 n, q^{2}\right)$. This intersects $H\left(2 n, q^{2}\right)$ in a cone with vertex $\pi_{1}^{\prime}$ and base a four-dimensional hermitian variety $H\left(4, q^{2}\right)$. The points of $\pi_{1}^{\prime \perp} \cap \mathcal{O}$ are projected from $\pi_{1}^{\prime}$ onto a partial ovoid $\mathcal{O}^{\prime}$ of $H\left(4, q^{2}\right)$.

Since a maximal partial ovoid of $H\left(4, q^{2}\right)$ has at least $q^{2}+q+2$ points (Result $2.1(\mathrm{~d})), \mathcal{O}^{\prime}$ is not maximal. So there exists a point $R$ in $H\left(4, q^{2}\right)$ extending the partial ovoid $\mathcal{O}^{\prime}$. Then the points of $\left\langle\pi_{1}^{\prime}, R\right\rangle \backslash \pi_{1}^{\prime}$ are not collinear with a point of $\pi_{1}^{\prime \perp} \cap \mathcal{O}$. Since $\mathcal{O}$ is a maximal partial ovoid, they all must be collinear with one of the, at most $q-1$, points of $\mathcal{O} \backslash \pi_{1}^{\prime \perp}$.

Let $S \in \mathcal{O} \backslash \pi_{1}^{\prime \perp}$, then $S^{\perp}$ intersects $\pi_{1}^{\prime}$ in a hyperplane of $\pi_{1}^{\prime}$. So for such a point $S, S^{\perp}$ intersects $\left\langle\pi_{1}^{\prime}, R\right\rangle$ in a hyperplane. These, at most $q-1$, hyperplanes in $\left\langle\pi_{1}^{\prime}, R\right\rangle$ must cover all the points in $\left\langle\pi_{1}^{\prime}, R\right\rangle \backslash \pi_{1}^{\prime}$. This is impossible.

Theorem 2.3 A maximal partial ovoid of $H\left(2 n+1, q^{2}\right), n \geq 2$, contains at least $q^{2}+q+1$ points.

Proof Assume that $\mathcal{O}$ is a maximal partial ovoid of $H\left(2 n+1, q^{2}\right), n \geq 2$, of size at most $q^{2}+q$. We repeat the beginning of the proof of the preceding theorem. Every free generator $\pi$ contains an $(n-2)$-dimensional space $\pi_{1}^{\prime}$ lying in a pencil of $q^{2}+1$ hyperplanes $P^{\perp} \cap \pi, P \in \mathcal{O}$, which cover all the points of $\pi$. 
The polar space $\pi_{1}^{\prime \perp}$ of $\pi_{1}^{\prime}$ with respect to $H\left(2 n+1, q^{2}\right)$ intersects $H\left(2 n+1, q^{2}\right)$ in a cone with vertex $\pi_{1}^{\prime}$ and base a hermitian variety $H\left(3, q^{2}\right)$. Since $H\left(3, q^{2}\right), q$ even, has partial ovoids of size $q^{2}+1$ (Result 2.1(b)), and only the lower bound $q^{2}+1+4 q / 9$ is known on the size of maximal partial ovoids of $H\left(3, q^{2}\right), q$ odd, (Result 2.1(c)), a different approach has to be used than in the proof of the preceding theorem.

Consider the points of $\mathcal{O} \backslash \pi_{1}^{\prime \perp}$. There are at most $q-1$ such points $S$. The tangent hyperplane to $S \in \mathcal{O} \backslash \pi_{1}^{\prime \perp}$ intersects $\pi_{1}^{\prime}$ in a hyperplane of $\pi_{1}^{\prime}$. These, at most $q-1$, hyperplanes cannot cover all the points of $\pi_{1}^{\prime}$. Let $P$ be a point of $\pi_{1}^{\prime}$ not collinear with a point of $\mathcal{O} \backslash \pi_{1}^{\prime \perp}$, then $P^{\perp} \cap \mathcal{O}=\pi_{1}^{\prime \perp} \cap \mathcal{O}$. Project $P^{\perp} \cap \mathcal{O}$ from $P$ onto its quotient geometry $H\left(2 n-1, q^{2}\right)$. This gives a new partial ovoid $\mathcal{O}^{\prime}$ of $H\left(2 n-1, q^{2}\right)$.

Suppose that $\mathcal{O}^{\prime}$ is not a maximal partial ovoid of $H\left(2 n-1, q^{2}\right)$. Then there is a point $R$ of $H\left(2 n-1, q^{2}\right)$ extending $\mathcal{O}^{\prime}$ to a larger partial ovoid. Hence, no point of the set $P R \backslash\{P\}$ is collinear with a point of $\mathcal{O} \cap \pi_{1}^{\prime \perp}$. The polar hyperplane of a point of $\mathcal{O} \backslash \pi_{1}^{\prime \perp}$ covers only one point of $P R \backslash\{P\}$. So at least $q^{2}-q+1$ points of $P R \backslash\{P\}$ are not collinear with a point of the maximal partial ovoid $\mathcal{O}$. This is a contradiction.

So, $\mathcal{O}^{\prime}$ indeed is a maximal partial ovoid of $H\left(2 n-1, q^{2}\right)$. Proceeding in this way, a maximal partial ovoid $\mathcal{O}^{\prime \prime}$ of $H\left(5, q^{2}\right)$ of size at most $q^{2}+q$ is obtained.

Consider this maximal partial ovoid $\mathcal{O}^{\prime \prime}$ of $H\left(5, q^{2}\right)$ of size at most $q^{2}+q$. The proof of the preceding theorem shows that every free generator $\pi$ to $\mathcal{O}^{\prime \prime}$ contains a point $P$ lying on $q^{2}+1$ lines $S^{\perp} \cap \pi, S \in \mathcal{O}^{\prime \prime}$, covering all the points of $\pi$. As $\left|\mathcal{O}^{\prime \prime}\right| \leq q^{2}+q$, the point $P$ is uniquely determined by $\pi$. We call a point $P \in H\left(5, q^{2}\right) \backslash \mathcal{O}$ a special point, if it is perpendicular to at least $q^{2}+1$ points of $\mathcal{O}$. Then every free plane has a unique special point. A special point $P$ lies on $(q+1)\left(q^{3}+1\right)$ planes of $H\left(5, q^{2}\right)$ but at least $\left|P^{\perp} \cap \mathcal{O}\right|(q+1) \geq\left(q^{2}+1\right)(q+1)$ contain a point of $\mathcal{O}$. Thus the number of free planes on a special point is at most $\left(q^{3}-q^{2}\right)(q+1)$. The total number of free planes is $\left(q^{5}+1-|\mathcal{O}|\right)(q+1)\left(q^{3}+1\right)$. Hence the number of special points is at least

$$
\frac{\left(q^{5}-q^{2}-q+1\right)(q+1)\left(q^{3}+1\right)}{\left(q^{3}-q^{2}\right)(q+1)}>q^{5}+q^{4}+q^{3}+q^{2} .
$$

As partial ovoids of $H\left(5, q^{2}\right)$ have less than $q^{5}+1$ points (that is the size of an ovoid), it follows that we find two special points $P$ and $Q$ that are perpendicular. As the line $P Q$ lies on $q+1$ planes of $H\left(5, q^{2}\right)$, at most $q+1$ points of $\mathcal{O}$ are perpendicular to $P$ and $Q$. As $P$ and $Q$ are special, it follows that $|\mathcal{O}| \geq 2\left(q^{2}+1\right)-(q+1)=2 q^{2}-q+1$. But $|\mathcal{O}| \leq q^{2}+q$, a contradiction.

\section{Large partial ovoids}

It is known that every partial ovoid of $H\left(3, q^{2}\right)$ with more than $q^{3}+1-q$ points can be extended to an ovoid. We will develop an analogous result for $H\left(5, q^{2}\right)$. It is known that $H\left(4, q^{2}\right)$ has no ovoids [17]. An upper bound on the size of partial ovoids in $H\left(4, q^{2}\right)$ is known [11]. We will improve on this result. We first mention two results.

\section{Result 3.1}

(a) Every partial ovoid of $H\left(3, q^{2}\right)$ of size larger than $q^{3}-q+1$ is extendable in a unique way to an ovoid of $H\left(3, q^{2}\right)$.

(b) A partial ovoid of $H\left(4, q^{2}\right)$ has size smaller than $q^{5}-(4 q-1) / 3$. 
Proof

(a) This was proven by Klein and Metsch in the dual setting of $Q^{-}(5, q)$ [13]. They proved that a partial spread of $Q^{-}(5, q)$ of size larger than $q^{3}-q+1$ is extendable to a spread of $Q^{-}(5, q)$.

The uniqueness of this extension to a spread follows from the fact that $\mathcal{S}$ is extended by less than $q+1$ lines to a spread.

(b) See [11].

Our next theorem improves Result 3.1(b). We mention that no large partial ovoids of $H\left(4, q^{2}\right)$ are known to us. The largest example we know about exists in characteristic three, so for $q=3^{h}$, and has size $q^{4}+1$ (Mazzocca et al. [14]).

Theorem 3.2 A partial ovoid of $H\left(4, q^{2}\right)$ has at most $q^{5}-q^{4}+q^{3}+1$ points.

Proof Let $\mathcal{O}$ be a partial ovoid of $H\left(4, q^{2}\right)$ with $s=q^{5}+1-\delta \geq 2$ points. Then there are $\delta\left(q^{3}+1\right)$ free generators, that is generators missing $\mathcal{O}$. We count the number of ordered triples $\left(P_{1}, P_{2}, g\right)$, where $P_{1}$ and $P_{2}$ are different points of $\mathcal{O}$ and $g$ is a free generator, such that $P_{1}$ and $P_{2}$ are in $H\left(4, q^{2}\right)$ collinear with the same point of $g$.

For a free generator $g$, every point of $\mathcal{O}$ has a unique neighbour on $g$. So on the average, a point of $g$ has $A:=s /\left(q^{2}+1\right)$ neighbours in $\mathcal{O}$. It follows that the number of ordered pairs $\left(P_{1}, P_{2}\right)$ of different points of $\mathcal{O}$ with the same neighbour on $g$ is at least $A(A-1)\left(q^{2}+1\right)=s(A-1)$.

Thus, the total number of ordered triples is at least $\delta\left(q^{3}+1\right) s(A-1)$. It follows that there exist two different points $P_{1}, P_{2} \in \mathcal{O}$ that occur in at least

$$
\frac{\delta\left(q^{3}+1\right) s(A-1)}{s(s-1)}=\frac{\delta\left(q^{3}+1\right)(A-1)}{s-1}
$$

of these triples. Let $l$ be the line on $P_{1}$ and $P_{2}$ of $\mathrm{PG}\left(4, q^{2}\right)$. Then $\pi:=l^{\perp}$ is a plane meeting $H\left(4, q^{2}\right)$ in a hermitian curve $H\left(2, q^{2}\right)$. No point of $\mathcal{O}$ lies in $\pi$. Denote by $k$ the number of points of $\mathcal{O}$ on $l$.

Next count the number of pairs $(X, Y)$, with $X$ in $H\left(2, q^{2}\right)=H\left(4, q^{2}\right) \cap \pi$ and $Y$ a point of $\mathcal{O}$ but not on $l$. By first choosing $X$, we see that the number of pairs is the number of non-free generators that meet $\pi$, but not $l \cap \mathcal{O}$. The number of generators meeting $\pi$ is $\left(q^{3}+1\right)\left(q^{3}+1\right)$. Exactly $\left(q^{3}+1\right) k$ of these generators meet $\mathcal{O} \cap l$. The choice of $P_{1}$ and $P_{2}$ yields that at least $\delta\left(q^{3}+1\right)(A-1) /(s-1)$ of these generators are free generators. Thus, the number of pairs $(X, Y)$ we are counting is at most

$$
\left(q^{3}+1\right)^{2}-\left(q^{3}+1\right) k-\frac{\delta\left(q^{3}+1\right)(A-1)}{s-1} .
$$

Now we find a lower bound. For a point $Y \in \mathcal{O} \backslash l$, the line $Y^{\perp} \cap \pi$ is either a tangent line of $H\left(2, q^{2}\right)=\pi \cap H\left(4, q^{2}\right)$ or a secant line of $H\left(4, q^{2}\right)$. So such a point $Y$ yields either one or $q+1$ pairs $(X, Y)$.

The first case occurs if and only if $Y$ lies on a generator that meets $l$ and $\pi$. There are $\left(q^{3}+1\right)(q+1)$ such generators but $\left(q^{3}+1\right) k$ of these meet $\mathcal{O}$ in a point on $l$. So the first case can occur at most $\left(q^{3}+1\right)(q+1-k)$ times. It follows that the total number of pairs is at least

$$
\begin{aligned}
& \left(q^{3}+1\right)(q+1-k) \cdot 1+\left(s-k-\left(q^{3}+1\right)(q+1-k)\right)(q+1) \\
& \quad=(s-k)(q+1)-\left(q^{3}+1\right)(q+1-k) q .
\end{aligned}
$$


Comparing the two bounds, we find

$$
\begin{aligned}
& (s-k)(q+1)-\left(q^{3}+1\right)(q+1-k) q \\
& \quad \leq\left(q^{3}+1\right)^{2}-\left(q^{3}+1\right) k-\frac{\delta\left(q^{3}+1\right)(A-1)}{s-1} .
\end{aligned}
$$

As $k \geq 2$, this remains true when $k$ is replaced by two. Doing this and replacing $A=s /\left(q^{2}+1\right)$ and $s=q^{5}+1-\delta$ gives

$$
\frac{q(q+1)\left(\delta-q^{4}+q^{3}\right)\left(q^{5}+q^{3}-\delta\right)}{\left(q^{2}+1\right)\left(q^{5}-\delta\right)} \geq 0 .
$$

Hence $\delta \geq q^{4}-q^{3}$.

Theorem 3.3 Every partial ovoid of $H\left(5, q^{2}\right)$ with $q^{5}+1-\delta$ points and $\delta \leq\left(q^{2}-\frac{1}{4} q+\right.$ 1) $/ \sqrt{2}$ is a subset of an ovoid.

Proof Let $\mathcal{O}$ be a maximal partial ovoid with $q^{5}+1-\delta$ points and $\delta>0$. We shall show that $\delta>\frac{1}{\sqrt{2}}\left(q^{2}-\frac{1}{4} q+1\right)$.

We call a point $P \in H\left(5, q^{2}\right) \backslash \mathcal{O}$ big when $\left|P^{\perp} \cap \mathcal{O}\right|>q^{3}+1-q$; otherwise it is called small. We call a plane of $H\left(5, q^{2}\right)$ free, if it misses $\mathcal{O}$. A totally isotropic line will be called free, if all totally isotropic planes on it are free planes.

We first show that $\left|P^{\perp} \cap \mathcal{O}\right| \geq q^{3}+1-\delta$ for every point $P \in H\left(5, q^{2}\right) \backslash \mathcal{O}$. For this, we use that the maximality of $\mathcal{O}$ implies that $P$ lies on a line $l$ of $H\left(5, q^{2}\right)$ meeting $\mathcal{O}$ in a point $Q$. Then every point of $\mathcal{O} \backslash\{Q\}$ is connected to exactly one point of $l \backslash\{Q\}$ by a line of $H\left(5, q^{2}\right)$. As each point of $l$ lies on at most $q^{3}+1$ lines meeting the partial ovoid $\mathcal{O}$ (because in the quotient geometry of such a point $P$ we see a hermitian variety $H\left(3, q^{2}\right)$ and partial ovoids of a hermitian variety $H\left(3, q^{2}\right)$ have at most $q^{3}+1$ points $)$, it follows that $P$ is connected to at least $|\mathcal{O}|-\left(q^{2}-1\right) q^{3}=q^{3}+1-\delta$ points of $\mathcal{O}$ by a line of $H\left(5, q^{2}\right)$.

For the rest of the proof, consider a free plane $\pi$. We show for each point $P$ of $\pi$ that the number of free planes on $P$ that meet $\pi$ in a line is at most $q+\delta-1$. Each point of $P^{\perp} \cap \mathcal{O}$ lies in a unique totally isotropic plane that meets $\pi$ in a line, and this line contains $P$. Since the number of totally isotropic planes on $P$ that meet $\pi$ in a line is $\left(q^{2}+1\right) q$, it follows that at most $\left(q^{2}+1\right) q-\left(q^{3}+1-\delta\right)=q-1+\delta$ of these are free planes.

We use the following notations:

- $f_{p}$ is the number of free planes that meet $\pi$ in a line.

- $b$ is the number of big points of $\pi$.

- $s$ is the number of small points of $\pi$.

- $f_{l}$ is the number of free lines in $\pi$.

- $z$ is the smallest number of small points on a free line of $\pi$.

A point is perpendicular to at most $q^{3}+1$ points of $\mathcal{O}$ with equality if and only if every generator on $P$ meets $\mathcal{O}$. Thus, the points of the free plane $\pi$ are perpendicular to at most $q^{3}$ points of $\mathcal{O}$, since they lie in a free plane; also the $s$ small points of $\pi$ are perpendicular to at most $q^{3}-q+1$ points of $\mathcal{O}$. As each point of $\mathcal{O}$ is perpendicular to $q^{2}+1$ points of $\pi$, we conclude that

$$
|\mathcal{O}|\left(q^{2}+1\right) \leq b q^{3}+s\left(q^{3}+1-q\right)=\left(q^{4}+q^{2}+1\right)\left(q^{3}+1-q\right)+b(q-1)
$$


and this gives

$$
\frac{q^{5}-q^{4}+q-\delta\left(q^{2}+1\right)}{q-1} \leq b .
$$

Consider a big point $P$ of $\pi$. In the quotient geometry of $P$, we see a hermitian variety $H\left(3, q^{2}\right)$. Also $P^{\perp} \cap \mathcal{O}$ gives rise to a partial ovoid of this $H\left(3, q^{2}\right)$. As $q^{3}-q+1<\left|P^{\perp} \cap \mathcal{O}\right|<q^{3}+1$, this partial ovoid can be extended uniquely to an ovoid, see Result 3.1(a). As $\pi$ is a free plane on $P$, it follows that a unique line of $\pi$ on $P$ has the property that every totally isotropic plane on that line is missing $\mathcal{O}$. This shows that the free lines of $\pi$ cover every big point of $\pi$ exactly once. As a free line of $\pi$ has at most $q^{2}+1-z$ big points, we conclude that

$$
b \leq f_{l}\left(q^{2}+1-z\right) \text {. }
$$

As each point of $\mathcal{O}$ is perpendicular to exactly one line of $\pi$, then exactly $|\mathcal{O}|$ of the planes that meet $\pi$ in a line are not free. Hence, the number of free planes that meet $\pi$ in a line is

$$
f_{p}=\left(q^{4}+q^{2}+1\right) q-|\mathcal{O}|=q^{3}+q-1+\delta .
$$

As every free line of $\pi$ lies in $q$ of these free planes, we have

$$
f_{l q} \leq f_{p} .
$$

We have seen above that every point of $\pi$ lies on at most $q-1+\delta$ free planes that meet $\pi$ in a line. This implies that every point of $\pi$ can lie on at most $(q-1+\delta) / q$ free lines of $\pi$. Also, as we have seen above, a big point of $\pi$ lies on a unique free line. Consider a free line $l$ of $\pi$ containing exactly $z$ small points. Then each of the $f_{l}-1$ other free lines contains one of these $z$ small points and hence

$$
f_{l}-1 \leq z\left(\frac{q-1+\delta}{q}-1\right) \Rightarrow f_{l} \leq \frac{q+z(\delta-1)}{q} .
$$

Comparing the lower bound (2) for $f_{l}$ with the upper bound (4), using (3), gives

$$
\frac{b}{q^{2}+1-z} \leq \frac{q^{3}+q-1+\delta}{q} .
$$

This gives

$$
z \leq z_{0}:=q^{2}+1-\frac{b q}{q^{3}+q-1+\delta} .
$$

Comparing the lower bound (2) for $f_{l}$ with the upper bound (5) gives

$$
b q \leq\left(q^{2}+1-z\right)(q+z(\delta-1)) .
$$

We conclude that $\delta \neq 1$, since otherwise $b \leq q^{2}+1-z$ contradicting (1). Hence $\delta \geq 2$.

Case 1 Here we consider the case when $q \geq 5$. We may assume that $\delta \leq q^{2}$. Then (1) and (6) imply that $z_{0} \leq \frac{1}{2}\left(q^{2}+1-q\right)$. Since $\delta \geq 2$, the right-hand side of (7), considered as a function of $z$, is monotone increasing for $z \leq \frac{1}{2}\left(q^{2}+1-q\right)$. Thus (7) 
remains true, if we replace $z$ by $z_{0}$. We do this and estimate $b$ in the formula of $z_{0}$ using (1). This results in

$$
\begin{aligned}
0 \leq & \frac{\left(q^{5}-q^{4}+q-\delta q^{2}-\delta\right) q^{2}}{(q-1)^{2}\left(q^{3}+q-1+\delta\right)^{2}} \\
& \cdot\left(q \delta-\delta-3 \delta q^{2}+2 \delta^{2} q^{2}-q \delta^{2}+\delta^{2}+q^{5}-q^{4}+q^{3}+q^{2}-q^{6}\right) .
\end{aligned}
$$

Hence $f(\delta) \geq 0$ where

$$
f(x)=q x-x-3 x q^{2}+2 x^{2} q^{2}-q x^{2}+x^{2}+q^{5}-q^{4}+q^{3}+q^{2}-q^{6} .
$$

The left-hand side is a polynomial of degree two in $x$ with positive leading coefficient. As $f(0)<0$ and $f\left(\left(q^{2}-\frac{1}{4} q+1\right) / \sqrt{2}\right)<0$, it follows that $\delta>\left(q^{2}-\frac{1}{4} q+1\right) / \sqrt{2}$.

Case 2 Here we consider the case when $q \in\{2,3,4\}$. Each case can be easily done by hand. We demonstrate the case $q=2$ and leave $q \in\{3,4\}$ to the reader. For $q=2$, we have to prove that $\delta \geq 3$. As we already know that $\delta \geq 2$, we just have to exclude $\delta=2$. Assume that $\delta=2$. Then (1), (6) and (7) read as follows

$$
8 \leq b, \quad z \leq 5-\frac{2 b}{11}, \quad 2 b \leq(5-z)(2+z) .
$$

Hence, $16 \leq(5-z)(2+z)$ for some $z \leq 3$. This is clearly impossible.

The bounds on $H\left(4, q^{2}\right)$ and $H\left(5, q^{2}\right)$ imply bounds on partial ovoids of $H\left(d, q^{2}\right)$ for $d \geq 6$, at least in the case when $d$ is even. For odd $d$, the situation is more complicated as we explain below.

Lemma 3.4 Denote by $o\left(H\left(d, q^{2}\right)\right)$ the largest cardinality of a partial ovoid of $H\left(d, q^{2}\right)$. Then for $d \geq 5$,

$$
o\left(H\left(d, q^{2}\right)\right) \leq q^{2} \cdot o\left(H\left(d-2, q^{2}\right)\right)-q^{2}+1 .
$$

Proof Consider a line $l$ of $H\left(d, q^{2}\right)$ meeting $\mathcal{O}$ in a point $P$. Then every point of $\mathcal{O} \backslash\{P\}$ is perpendicular to a unique point of $l \backslash\{P\}$. As the points of $\mathcal{O}$ perpendicular to the point $X \in l \backslash\{P\}$ form a partial ovoid in the hermitian polar space $H\left(d-2, q^{2}\right)$ seen in the quotient geometry at $X$, the result follows.

It is not known whether $H\left(5, q^{2}\right)$ has an ovoid. If ever it can be proven that ovoids do not exist, then the preceding lemma in conjunction with Theorem 3.3 gives an upper bound on the size of a maximal partial ovoid of $H\left(2 n+1, q^{2}\right), n \geq 2$.

By Theorem 3.2, a partial ovoid of $H\left(4, q^{2}\right)$ has at most $q^{5}-q^{4}+\bar{q}^{3}+1$ points. Applying Lemma 3.4 thus shows that a partial ovoid of $H\left(2 n, q^{2}\right), n \geq 2$, has at most $q^{2 n+1}+1-q^{2(n-2)}\left(q^{4}-q^{3}\right)$ points. However, the following lemma gives a better recursive bound than Lemma 3.4.

\section{Lemma 3.5}

$$
\begin{aligned}
& \left|H\left(2 n-2, q^{2}\right)\right| \cdot\left(o\left(H\left(2 n-2, q^{2}\right)\right)-2\right) \\
& \quad \geq\left(o\left(H\left(2 n, q^{2}\right)\right)-2\right)\left|H\left(2 n-3, q^{2}\right)\right|-(q-1) \cdot o\left(H\left(2 n-2, q^{2}\right)\right) \cdot q^{2 n-3} .
\end{aligned}
$$

Proof Suppose that $\mathcal{O}$ is a partial ovoid of $H\left(2 n, q^{2}\right)$ of size $o\left(H\left(2 n, q^{2}\right)\right)$. Consider a secant line $l$ of $H\left(2 n, q^{2}\right)$ meeting $\mathcal{O}$ in $k \geq 2$ points, and put $\pi=l^{\perp}$. 
We count the number of pairs $(X, Y)$, with $X$ in $H\left(2 n-2, q^{2}\right)=H\left(2 n, q^{2}\right) \cap \pi$ and $Y$ a point of $\mathcal{O}$ but not on $l$. By first choosing $X$, we find the upper bound $\left|H\left(2 n-2, q^{2}\right)\right| \cdot\left(o\left(H\left(2 n-2, q^{2}\right)\right)-k\right)$, since each of the points of $H\left(2 n-2, q^{2}\right)$ is perpendicular to at most $o\left(H\left(2 n-2, q^{2}\right)\right)$ points of $\mathcal{O}$.

Now we first choose $Y$. If $Y$ is not perpendicular to one of the points of $l \cap H\left(2 n, q^{2}\right)$, then $\langle l, Y\rangle$ meets $H\left(2 n, q^{2}\right)$ in a hermitian curve and thus $Y^{\perp} \cap \pi$ meets $H\left(2 n, q^{2}\right)$ in a hermitian polar space $H\left(2 n-3, q^{2}\right)$. If $Y$ is perpendicular to one of the points of $l \cap H\left(2 n, q^{2}\right)$ (this must be one of the $q+1-k$ points of $l \cap H\left(2 n, q^{2}\right)$ not in $\left.\mathcal{O}\right)$, then $Y^{\perp} \cap \pi$ meets $H\left(2 n, q^{2}\right)$ in a cone with a point vertex over a $H\left(2 n-4, q^{2}\right)$ as base. In the first case, $Y$ occurs in $\left|H\left(2 n-3, q^{2}\right)\right|$ pairs $(X, Y)$ but in the second case it occurs only in $1+q^{2}\left|H\left(2 n-4, q^{2}\right)\right|$ such pairs; this is $q^{2 n-3}$ less than in the first case. The second case can occur for at most $(q+1-k) o\left(H\left(2 n-2, q^{2}\right)\right)$ points $Y$, since each of the $q+1-k$ points of $l \cap H\left(2 n, q^{2}\right)$ that are not in $\mathcal{O}$ is perpendicular to at most $o\left(H\left(2 n-2, q^{2}\right)\right)$ points of $\mathcal{O}$. Thus we find

$$
\left(o\left(H\left(2 n, q^{2}\right)\right)-k\right)\left|H\left(2 n-3, q^{2}\right)\right|-(q+1-k) \cdot o\left(H\left(2 n-2, q^{2}\right)\right) \cdot q^{2 n-3}
$$

as a lower bound on the number of pairs. Comparing the upper and lower bound, we find an inequality, which gets stronger for large $k$. For the smallest possible value $k=2$, we find the statement.

Applying this with $n=3$ using that partial ovoids of $H\left(4, q^{2}\right)$ have at most $q^{5}+1-d_{2}$ points with $d_{2}=q^{4}-q^{3}$, we find that partial ovoids of $H\left(6, q^{2}\right)$ have at most $q^{7}+1-d_{3}$ points with $d_{3}=q^{6}-q^{5}+q^{3}-1$ points. For $n \geq 4$, we find recursively upper bounds $q^{2 n+1}+1-d_{n}$ for partial ovoids of $H\left(2 n+1, q^{2}\right)$ with $d_{n}=q^{2} d_{n-1}+q^{3}$; we omit the technical calculations. This leads to the following corollary.

Corollary 3.6 A partial ovoid of the hermitian polar space $H\left(2 n, q^{2}\right), n \geq 3$, has at most

$$
q^{2 n+1}+1-q^{2(n-3)}\left(q^{6}-q^{5}-1\right)-q^{3} \cdot \frac{q^{2(n-2)}-1}{q^{2}-1}
$$

points.

\section{Partial spreads}

In ref. [18], Thas proved the upper bound $q^{2 n+1}-q^{n+1}+q^{n}+1$ for partial spreads of $H\left(2 n+1, q^{2}\right), n \geq 1$ odd. In this section, we first improve this result and then prove a similar result for $H\left(2 n+1, q^{2}\right), n \geq 2$ even. To our knowledge, a better bound is known only for $H\left(5, q^{2}\right)$, see [7], where the exact upper bound $q^{3}+1$ was found by De Beule and Metsch for the size of partial spreads of $H\left(5, q^{2}\right)$. A main ingredient for our argument is the following beautiful property of hermitian spaces proven by Thas [18].

Result 4.1 Let $\pi_{1}, \pi_{2}$ and $\pi$ be mutually skew generators of $H\left(2 n+1, q^{2}\right)$. Then the points of $\pi$ that lie on a line of $H\left(2 n+1, q^{2}\right)$ meeting $\pi_{1}$ and $\pi_{2}$ form a hermitian variety $H\left(n, q^{2}\right)$ in $\pi$.

Theorem 4.2 Let $\mathcal{S}$ be a partial spread of $H\left(2 n+1, q^{2}\right)$.

(a) If $n=1$, then $|\mathcal{S}| \leq \frac{1}{2}\left(q^{3}+q+2\right)$.

(b) If $n \geq 3$ is odd, then $|\mathcal{S}| \leq q^{2 n+1}-q^{(3 n+4) / 2}+q^{(3 n+3) / 2}$. 
Proof For $i \in \mathbb{N}$, put $T_{i}:=\left(q^{2 i+2}-1\right) /\left(q^{2}-1\right)$. Suppose that $\mathcal{S}$ is a partial spread of $H\left(2 n+1, q^{2}\right)$ and put $|\mathcal{S}|=q^{2 n+1}+1-\delta$. Then the number of points of $H\left(2 n+1, q^{2}\right)$ that are not covered by generators of $\mathcal{S}$ is $h:=\delta T_{n}$. We call these points the holes.

Consider the triples $\left(S_{1}, S_{2}, P\right)$, where $S_{1}$ and $S_{2}$ are different elements of $\mathcal{S}$ and $P$ is a hole. We shall estimate how many of these triples have the property that the unique line of $\mathrm{PG}\left(2 n+1, q^{2}\right)$ on $P$ that meets $S_{1}$ and $S_{2}$ (that is, the line $\left\langle P, S_{1}\right\rangle \cap\left\langle P, S_{2}\right\rangle$ ) belongs to $H\left(2 n+1, q^{2}\right)$.

To do so, we consider a hole $P$. Then $P$ lies on $\left|H\left(2 n-1, q^{2}\right)\right|$ lines of $H\left(2 n+1, q^{2}\right)$. The number of points on these lines that are covered by an element of $\mathcal{S}$ is $|\mathcal{S}| T_{n-1}$. If $x_{i}, i=1, \ldots,\left|H\left(2 n-1, q^{2}\right)\right|$, is the number of points on the $i$ th line on $P$ that are covered by an element of $\mathcal{S}$, then we have $\sum x_{i}=|\mathcal{S}| T_{n-1}$ and hence

$$
\begin{aligned}
\sum x_{i}\left(x_{i}-1\right) & \geq \frac{|\mathcal{S}| T_{n-1}}{\left|H\left(2 n-1, q^{2}\right)\right|}\left(\frac{|\mathcal{S}| T_{n-1}}{\left|H\left(2 n-1, q^{2}\right)\right|}-1\right)\left|H\left(2 n-1, q^{2}\right)\right| \\
& =|\mathcal{S}| T_{n-1}\left(\frac{|\mathcal{S}| T_{n-1}}{\left|H\left(2 n-1, q^{2}\right)\right|}-1\right) .
\end{aligned}
$$

Since the number of holes is $\delta T_{n}$, we find a lower bound on the number of triples considered above. Now we choose a pair $\left(S_{1}, S_{2}\right)$ of distinct spread elements such that the number $\alpha$ of considered triples $\left(S_{1}, S_{2}, P\right)$ is as large as possible. We find that $\alpha \geq \alpha_{0}$ with $\alpha_{0}$ defined by

$$
\begin{aligned}
|\mathcal{S}|(|\mathcal{S}|-1) \alpha_{0} & =\delta T_{n}|\mathcal{S}| T_{n-1}\left(\frac{|\mathcal{S}| T_{n-1}}{\left|H\left(2 n-1, q^{2}\right)\right|}-1\right) \\
& =|\mathcal{S}| \delta T_{n} T_{n-1} \cdot \frac{|\mathcal{S}|-q^{2 n-1}-1}{q^{2 n-1}+1} .
\end{aligned}
$$

Consider all lines of $H\left(2 n+1, q^{2}\right)$ that meet $S_{1}$ and $S_{2}$. There are $T_{n} T_{n-1}$ such lines. Thus, the number of points outside of $S_{1}$ and $S_{2}$ that lie on a line of $H\left(2 n+1, q^{2}\right)$ meeting $S_{1}$ and $S_{2}$ is $T_{n} T_{n-1}\left(q^{2}-1\right)$. Each $S \in \mathcal{S} \backslash\left\{S_{1}, S_{2}\right\}$ contains $\left|H\left(n, q^{2}\right)\right|$ of these points by Result 4.1. Also, at least $\alpha_{0}$ of these points are holes. Hence

$$
(|\mathcal{S}|-2)\left|H\left(n, q^{2}\right)\right|+\alpha_{0} \leq T_{n} T_{n-1}\left(q^{2}-1\right) .
$$

Hence

$$
(|\mathcal{S}|-1)(|\mathcal{S}|-2)\left|H\left(n, q^{2}\right)\right|+\delta T_{n} T_{n-1} \frac{|\mathcal{S}|-q^{2 n-1}-1}{q^{2 n-1}+1} \leq T_{n} T_{n-1}\left(q^{2}-1\right)(|\mathcal{S}|-1) .
$$

As $n$ is odd, we have $\left|H\left(n, q^{2}\right)\right|=\left(q^{n}+1\right)\left(q^{n+1}-1\right) /\left(q^{2}-1\right)$ and thus we find

$$
\begin{aligned}
& (|\mathcal{S}|-1)(|\mathcal{S}|-2)+\delta \frac{\left(q^{n}-1\right)\left(q^{n+1}+1\right)}{q^{2}-1} \cdot \frac{|\mathcal{S}|-q^{2 n-1}-1}{q^{2 n-1}+1} \\
& \quad \leq\left(q^{n}-1\right)\left(q^{n+1}+1\right)(|\mathcal{S}|-1) .
\end{aligned}
$$

Replace $|\mathcal{S}|-2$ by $q^{2 n+1}-1-\delta$ to find

$$
(|\mathcal{S}|-1)\left(q^{n+1}-q^{n}-\delta\right)+\delta \frac{\left(q^{n}-1\right)\left(q^{n+1}+1\right)}{q^{2}-1} \cdot \frac{|\mathcal{S}|-q^{2 n-1}-1}{q^{2 n-1}+1} \leq 0 .
$$

For $n=1$, we multiply this by $(q+1)^{2}$ and find after simplification

$$
\left(q^{3}-2 \delta-q\right)\left(q^{3}+q^{2}-\delta\right) q \leq 0 .
$$


Hence for $n=1$, we have $\delta \geq \frac{1}{2}\left(q^{3}-q\right)$, that is $|\mathcal{S}| \leq \frac{1}{2}\left(q^{3}+q+2\right)$. For odd $n \geq 3$, we multiply by $\left(q^{2}-1\right)\left(q^{2 n-1}+1\right) / q^{2}$ and find

$$
\begin{aligned}
& \delta^{2}\left(q^{n-2}-1\right)\left(q^{n-1}+1\right)+\delta\left(q^{2}-1\right) q^{n-2}\left(q^{n}-q^{n-1}+1\right)\left(2 q^{n}+q-1\right) \\
& \quad \geq q^{3 n-1}(q-1)\left(q^{2}-1\right)\left(q^{2 n-1}+1\right) .
\end{aligned}
$$

It can be verified that this implies that $\delta \geq q^{(3 n+4) / 2}-q^{(3 n+3) / 2}+1$.

Remark 4.3 The argument gives a slightly better bound for $\delta$ but to avoid presenting a complicated lower bound on $\delta$, we opted for this more elegant bound on $\delta$.

Remark 4.4 An interesting feature of the upper bound $\left(q^{3}+q+2\right) / 2$ on the size of partial spreads of $H\left(3, q^{2}\right)$ is that this bound is sharp for $q=2$ and $q=3$. For $q=2$, Dye [9] constructed a maximal partial spread of size 6 on $H(3,4)$ by constructing a maximal partial ovoid of size 6 on $Q^{-}(5, q)$.

For $q=3$, Ebert and Hirschfeld [10] proved by an exhaustive computer search that a maximal partial spread on $H(3,9)$ has size at most 16 . Their computer search showed many properties of this maximal partial spread $\mathcal{S}$ of size 16 . It is linked to the Kummer surface. Every line of $H(3,9)$ not in $\mathcal{S}$ intersects either four or ten lines in $\mathcal{S}$. There are 16 lines not in $\mathcal{S}$ intersected by ten lines in $\mathcal{S}$; these latter 16 lines form themselves a maximal partial spread of size 16 of $H(3,9)$.

These properties which arose from the computer search can be proven theoretically by our arguments. We do this for general $q$. Suppose that $\mathcal{S}$ is a partial spread of $H\left(3, q^{2}\right)$ of size $\frac{1}{2}\left(q^{3}+q+2\right)$. Then there is equality in all the calculations of the proof of Theorem 4.2. In particular, there is equality in (8), so $x_{i}=|\mathcal{S} V|(q+1)=\frac{1}{2}\left(q^{2}-q+2\right)$, $i=1, \ldots, q+1$. We conclude that if a line contains a hole, then it intersects $\frac{1}{2}\left(q^{2}-q+2\right)$ lines of $\mathcal{S}$.

Consider a point $P$ on a line $l$ of $\mathcal{S}$. Then $P$ lies on $q$ further lines of $H\left(3, q^{2}\right)$. If such a line contains a hole, then it meets $\frac{1}{2}\left(q^{2}-q\right)$ further lines of $\mathcal{S}$. Otherwise it meets $q^{2}$ further lines of $\mathcal{S}$. As $|\mathcal{S}|-1=\frac{1}{2}\left(q^{3}+q\right)$, it readily follows that exactly one of the $q$ lines on $P$ does not contain a hole. Hence, if $\mathcal{S}^{\prime}$ denotes the set of all lines of $H\left(3, q^{2}\right)$ which do not lie in $\mathcal{S}$ and which do not contain a hole, the lines of $\mathcal{S}^{\prime}$ are mutually skew and cover the same points as the lines of $\mathcal{S}$. Hence $\left|\mathcal{S}^{\prime}\right|=|\mathcal{S}|=\frac{1}{2}\left(q^{3}+q+2\right)$. In other words, $\mathcal{S}^{\prime}$ is a second partial spread with the same number of lines and covering the same points as $\mathcal{S}$. Also $\mathcal{S} \cap \mathcal{S}^{\prime}=\emptyset$.

Consider two different lines $l_{1}, l_{2} \in \mathcal{S}$. Then $H\left(3, q^{2}\right)$ has $q^{2}+1$ lines $h_{i}, i=$ $1, \ldots, q^{2}+1$, meeting $l_{1}$ and $l_{2}$ and these lines are mutually skew. If a line $h_{i}$ lies in $\mathcal{S}^{\prime}$, then it meets $q^{2}+1$ lines of $\mathcal{S}$, and otherwise it meets $\frac{1}{2}\left(q^{2}-q+2\right)$ lines of $\mathcal{S}$. On the other hand, Result 4.1 says that every line $l_{3} \in \mathcal{S} \backslash\left\{l_{1}, l_{2}\right\}$ meets exactly $q+1$ of the lines $h_{i}$. Hence, if $\lambda$ is the number of lines $h_{i}$ that belong to $\mathcal{S}^{\prime}$, then

$$
2\left(q^{2}+1\right)+(|\mathcal{S}|-2)(q+1)=\lambda\left(q^{2}+1\right)+\left(q^{2}+1-\lambda\right) \cdot \frac{1}{2}\left(q^{2}-q+2\right) .
$$

Hence, $\lambda=2 q$. Clearly, every line of one of the partial spreads $\mathcal{S}$ and $\mathcal{S}^{\prime}$ meets precisely $q^{2}+1$ lines of the other partial spread. Thus, the incidence structure whose points are the lines of $\mathcal{S}$ and whose blocks are the lines of $\mathcal{S}^{\prime}$ and incidence defined as having non-trivial intersection, is a symmetric $2-(v, k, \lambda)$ design with $v=\frac{1}{2}\left(q^{3}+q+2\right)$ points, block size $k=q^{2}+1$, and $\lambda=2 q$. 
Corollary 4.5 If $H\left(3, q^{2}\right)$ possesses a partial spread of size $\frac{1}{2}\left(q^{3}+q+2\right)$, then there exists a symmetric $2-(v, k, \lambda)$ design with parameters $v=\frac{1}{2}\left(q^{3}+q+2\right), k=q^{2}+1$ and $\lambda=2 q$.

Notice that $k-\lambda=(q-1)^{2}$ is a perfect square, so no non-existence results on designs show that such a design might not exist. We mention also that there exist designs for $q=5$ and $q=7$ with these parameters!

The following result proves a similar bound for even $n$. We recall that for $n=2$ much more is known. It was shown in [7] that a partial spread of $H\left(5, q^{2}\right)$ has at most $q^{3}+1$ planes, and this upper bound is sharp.

Theorem 4.6 Let $\mathcal{S}$ be a partial spread of $H\left(2 n+1, q^{2}\right)$. If $n \geq 4$ is even, then $|\mathcal{S}|=q^{2 n+1}+1-\delta$ with

$$
\delta>-q^{n}(q-1)+\sqrt{q^{3 n+1}(q-1)+q^{2 n}(q-1)^{2}} .
$$

Proof For $i \in \mathbb{N}$, put $T_{i}:=\left(q^{2 i+2}-1\right) /\left(q^{2}-1\right)$. Suppose that $\mathcal{S}$ is a partial spread of $H\left(2 n+1, q^{2}\right)$ and put $|\mathcal{S}|=q^{2 n+1}+1-\delta$. Then the number of points of $H\left(2 n+1, q^{2}\right)$ that are not covered by generators of $\mathcal{S}$ is $h:=\delta T_{n}$. We call these points the holes.

Consider the triples $\left(S_{1}, S_{2}, P\right)$, where $S_{1}$ and $S_{2}$ are different elements of $\mathcal{S}$ and $P$ is a hole. We again estimate how many of these triples have the property that the unique line of $\operatorname{PG}\left(2 n+1, q^{2}\right)$ on $P$ that meets $S_{1}$ and $S_{2}$ belongs to $H\left(2 n+1, q^{2}\right)$.

Consider all lines of $H\left(2 n+1, q^{2}\right)$ that meet $S_{1}$ and $S_{2}$. There are $T_{n} T_{n-1}$ such lines. Thus the number of points outside of $S_{1}$ and $S_{2}$ that lie on a line of $H\left(2 n+1, q^{2}\right)$ meeting $S_{1}$ and $S_{2}$ is $T_{n} T_{n-1}\left(q^{2}-1\right)$. Each $S \in \mathcal{S} \backslash\left\{S_{1}, S_{2}\right\}$ contains $\left|H\left(n, q^{2}\right)\right|$ of these points by Result 4.1. Hence, if $h_{0}$ is the number of holes on these lines, then

$$
(|\mathcal{S}|-2)\left|H\left(n, q^{2}\right)\right|+h_{0}=T_{n} T_{n-1}\left(q^{2}-1\right) .
$$

In other words: for any two different elements $S_{1}$ and $S_{2}$ of $\mathcal{S}$, there exist exactly $h_{0}$ holes which lie on a line of $H\left(2 n+1, q^{2}\right)$ meeting $S_{1}$ and $S_{2}$.

Now fix an element $S_{1} \in \mathcal{S}$. We have seen that every second spread element $S_{2}$ gives rise to $h_{0}$ holes that lie on a line of $H\left(2 n+1, q^{2}\right)$ meeting $S_{1}$ and $S_{2}$. Clearly every hole $P$ can occur for at most $q^{2 n}-1$ different elements $S_{2}$, because the space $\left\langle P, P^{\perp} \cap S_{1}\right\rangle$ has dimension $n$. Hence

$$
h\left(q^{2 n}-1\right) \geq h_{0}(|\mathcal{S}|-1) .
$$

Replacing $h_{0}$, using (9), and replacing $h$ by $\delta T_{n}$ results in

$$
\delta T_{n} T_{n-1}\left(q^{2}-1\right) \geq\left[T_{n} T_{n-1}\left(q^{2}-1\right)-(|\mathcal{S}|-2) \cdot\left|H\left(n, q^{2}\right)\right|\right](|\mathcal{S}|-1) .
$$

This is divisible by $\left|H\left(n, q^{2}\right)\right|$. Dividing this term out and using $|\mathcal{S}|=q^{2 n+1}+1-\delta$, the resulting expression simplifies to

$$
\delta^{2}+2 \delta q^{n}(q-1)-\delta \geq q^{3 n+1}(q-1) .
$$

This implies that

$$
\delta^{2}+2 \delta q^{n}(q-1)>q^{3 n+1}(q-1)
$$

Solving this quadratic inequality for $\delta$ gives the desired bound on $\delta$. 


\section{Tables}

To present an overview of the current results on small or large maximal partial ovoids and maximal partial spreads in hermitian polar spaces, we collect the results in two tables. The similar results for the orthogonal and symplectic polar spaces are presented in [8].

In Table 1 , the results on $H\left(3, q^{2}\right)$ are proven in $[2,7,15]$. The results on $H\left(d, q^{2}\right)$, $d \geq 4$, arise from the present paper. We remark that the bounds for $H\left(3, q^{2}\right), q$ even, are sharp. Also the upper bound for $H\left(3, q^{2}\right), q$ odd, is sharp.

In Table 2, the lower bounds for $H\left(d, q^{2}\right), d \geq 5$, have been proven in Theorem 8.1 of [8]. The lower bound for $H\left(3, q^{2}\right)$ is proven in [10], and the lower bound for $H\left(4, q^{2}\right)$ in [16]. The upper bound $q^{3}+1$ for $H\left(5, q^{2}\right)$ was proven in [7] and this upper bound is sharp. The upper bound for $H\left(2 n, q^{2}\right), n \geq 2$, is just the size of a spread, whose existence is still open and one of the main problems in this field. The upper bounds for $H\left(2 n+1, q^{2}\right), n \geq 1$, have all been proven in the present paper.

Remark 5.1 Next to the upper bounds on the size of maximal partial ovoids in hermitian polar spaces, presented in Table 1, there are the important bounds of Blokhuis and Moorhouse [3]. For large values of $n$, these upper bounds of Blokhuis and Moorhouse are better than the bounds of Table 1. It is however difficult to make an exact comparison between the bounds of Table 1 and those of Blokhuis and Moorhouse. For this reason, we refer to [3] for the bounds of Blokhuis and Moorhouse.

Table 1 Bounds on maximal partial ovoids

\begin{tabular}{lll}
\hline Polar space & Lower bounds & Upper bounds \\
\hline$H\left(3, q^{2}\right), q$ even & $q^{2}+1$ & $q^{3}+1$ \\
$H\left(3, q^{2}\right), q$ odd & $q^{2}+1+\frac{4}{9} q$ & $q^{3}+1$ \\
$H\left(4, q^{2}\right)$ & $q^{2}+q+2$ & $q^{5}-q^{4}+q^{3}+1$ \\
$H\left(2 n, q^{2}\right), n \geq 3$ & $q^{2}+q+1$ & $q^{2 n+1}+1-q^{2(n-3)}\left(q^{6}-q^{5}-1\right)$ \\
$H\left(2 n+1, q^{2}\right), n \geq 2$ & $q^{2}+q+1$ & $-q^{3} \cdot \frac{q^{2(n-2)}-1}{q^{2}-1}$ \\
\hline
\end{tabular}

Table 2 Bounds on maximal partial spreads

\begin{tabular}{lll}
\hline Polar space & Lower bounds & Upper bounds \\
\hline$H\left(3, q^{2}\right)$ & $2 q+1$, for $q \leq 3$ & $\frac{1}{2}\left(q^{3}+q+2\right)$ \\
$H\left(4, q^{2}\right)$ & $2 q+2$, for $q \geq 4$ & \\
$H\left(5, q^{2}\right)$ & $q^{3}+q \sqrt{q}-\frac{1}{2} q$ & \\
$H\left(2 n, q^{2}\right), n \geq 3$ & $-\frac{3}{8} \sqrt{q}+\frac{7}{8}$ & $q^{5}+1$ \\
$H\left(2 n+1, q^{2}\right), n \geq 3, n$ odd & $q+1$ & $q^{3}+1$ \\
$H\left(2 n+1, q^{2}\right), n \geq 3, n$ even & $q^{3}+1$ & $q^{2 n+1}+1$ \\
& $q+1$ & $q^{2 n+1}-q^{(3 n+4) / 2}+q^{(3 n+3) / 2}$ \\
& $q+1$ & $q^{2 n+1}+1+q^{n}(q-1)$ \\
\hline
\end{tabular}


Acknowledgments (1) This research was done while the first author was visiting the Justus-LiebigUniversität Gießen, Germany. He thanks the Fund for Scientific Research-Flanders (Belgium) for a research grant. (2) This research was done while the fourth author was visiting the Justus-LiebigUniversität Gießen, Germany, with an Alexander von Humboldt Fellowship. The fourth author wishes to thank the Alexander von Humboldt Foundation for granting him this Fellowship.

\section{References}

1. Aguglia A, Cossidente A, Ebert GL (2003) Complete spans on Hermitian varieties. Des Codes Cryptogr 29(1-3):7-15

2. Aguglia A, Ebert GL, Luyckx D (2005) On partial ovoids of Hermitian surfaces. Bull Belg Math Soc Simon Stevin 12(5):641-650

3. Blokhuis A, Moorhouse GE (1995) Some p-ranks related to orthogonal spaces. J Algebraic Comb 4(4):295-316

4. Bose RC, Burton RC (1966) A characterization of flat spaces in a finite geometry and the uniqueness of the Hamming and the MacDonald codes. J Comb Theory 1:96-104

5. Bruen AA (1980) Blocking sets and skew subspaces of projective space. Can J Math 32(3):628-630

6. Cimráková M, De Winter S, Fack V, Storme L (2006) On the smallest maximal partial ovoids and spreads of the generalized quadrangles $W(q)$ and $Q(4, q)$. Eur $\mathrm{J}$ Comb, doi:10.1016/j.ejc.2006.11.001

7. De Beule J, Metsch K (2006) The maximum size of a partial spread in $H\left(5, q^{2}\right)$ is $q^{3}+1$. J Comb Theory Ser A, doi:10.1016/j.jcta.2006.08.005

8. De Beule J, Klein A, Metsch K, Storme L (2007) Partial ovoids and partial spreads in symplectic and orthogonal polar spaces. Eur J comb (submitted)

9. Dye RH (1992) Maximal sets of nonpolar points of quadrics and symplectic polarities over GF(2). Geom Dedicata 44(3):281-293

10. Ebert GL, Hirschfeld JWP (1999) Complete systems of lines on a Hermitian surface over a finite field. Des Codes Cryptogr 17(1-3):253-268

11. Govaerts P, Storme L, Van Maldeghem H (2002) On a particular class of minihypers and its applications. III. Applications. Eur J Comb 23(6):659-672

12. Hirschfeld JWP, Thas JA (1991) General Galois geometries. Oxford Mathematical Monographs. The Clarendon Press Oxford University Press, Oxford Science Publications, New York

13. Klein A, Metsch K (2005) New results on covers and partial spreads of polar spaces. Innov Incidence Geom 1:19-34

14. Mazzocca F, Polverino O, Storme L (2006) Blocking sets in PG $\left(r, q^{n}\right)$ (preprint)

15. Metsch K (2006) Small maximal partial ovoids of $H\left(3, q^{2}\right)$. Innov Incidence Geom 3:1-12

16. Metsch K, Storme L. Maximal partial ovoids and maximal partial spreads in hermitian generalized quadrangles. J Comb Des, doi:10.1002/jcd.20146

17. Payne SE, Thas JA (1984) Finite generalized quadrangles, volume 110 of research notes in mathematics. Pitman (Advanced Publishing Program), Boston, MA

18. Thas JA (1992) Old and new results on spreads and ovoids of finite classical polar spaces. In: Proceedings of Combinatorics '90 (Gaeta, 1990). Annals of discrete mathematics, vol 52. North-Holland, Amsterdam, pp 529-544

19. Thas JA (2001) Ovoids, spreads and $m$-systems of finite classical polar spaces. In: Surveys in combinatorics, 2001 (Sussex). London mathematical society lecture note series, vol 288. Cambridge University Press, Cambridge, pp 241-267 